\title{
Lithostar Modularis de Siemens: exposición de los resultados de los 1.000 primeros pacientes
}

\author{
A. Vega Vega*, L.E. Parra Muntaner**, D. García Alonso*, C.J. García Alonso*, \\ J.M. Rodríguez Lamelas*, A.D. Reboto Rodríguez* \\ *Servicio de Urología de la Obra Hospitalaria Nuestra Señora de Regla, León. \\ ** Servicio Urología del Hospital del Bierzo, Ponferrada, León \\ Actas Urol Esp 2005; 29 (7): 662-666
}

\section{RESUMEN}

\section{LITHOSTAR MODULARIS DE SIEMENS: EXPOSICION DE LOS RESULTADOS DE LOS 1.000 PRIMEROS PACIENTES}

Pacientes y Métodos: Exponemos los resultados de los 1.000 primeros tratamientos de pacientes con litiasis en el sistema urinario efectuados desde mayo 2000 a diciembre 2003 con Lithostar Modularis de Siemens en un intento de valorar la eficacia de este litotritor de nueva generación.

Resultados: 60,5 \% varones y 39,5\% mujeres. Edad media 53,5 años. La localización de la litiasis: derecha $44,1 \%$ e izquierda 55,9\%. Tamaño medio de las litiasis $1,22 \pm 0,74 \mathrm{~cm}$. El $59 \% \leq 1 \mathrm{~cm}$, el $27,6 \%$ entre 1 y $2 \mathrm{~cm}$ y el $13,4 \% \geq 2 \mathrm{~cm}$. La media de sesiones fue de 1,6 por paciente, existiendo diferencias en el número de sesiones en cuanto al tamaño, 1,3 en $\leq 1 \mathrm{~cm}$ y 2,01 sesiones en $>1 \mathrm{~cm}$, y a la localización, menor número en las litiasis en uréter lumbar y en cáliz superior. La tasa de pacientes libres de litiasis en una sola sesión fue del $71,2 \%$.

Conclusiones: las ventajas de este litotritor, además de los adelantos técnicos tanto en comodidad del paciente, localización de la litiasis y mejor calidad de la escopia, se deben sobre todo a su alta eficacia, con una fragmentación de la litiasis en pequeños fragmentos, facilitando su eliminación, consiguiendo una resolución sin restos de litiasis por encima del 90\%. Además disminuye la necesidad de maniobras complementarias y acorta el tiempo de tratamiento por la fácil eliminación de los restos fragmentados.

Palabras clave: Litotricia. Litiasis renal. ESWL.

\section{ABSTRACT}

\section{CLINICAL EXPERIENCE AND RESULTS OF ESWL TREATMENT FOR 1.000 CONSECUTIVE PATIENTS WITH THE SIEMENS LITHOSTAR MODULARIS}

Patients and Methods: We describe the outcome of the treatment of 1.000 consecutive patients treated since may 2000 to december 2003 with Siemens Lithostar Modularis to evaluate the efficacy of this new lithotripter

Results: $60.5 \%$ male and 39.5\% female's patients were treated. Average age of 53.5 years old. $44.1 \%$ of the urinary stones were located on the right side and $55.9 \%$ on the left. The mean size of the stones was $1,22 \pm 0,74 \mathrm{~cm}$ with $59 \%$ lesser than $1 \mathrm{~cm}, 27.6 \%$ between 1 and $2 \mathrm{~cm}$ and $13.4 \%$ bigger than $2 \mathrm{~cm}$. Mean number of sessions required were 1.6. There were differences in the number of sessions related to the size of the lithiasis, 1.3 session in the group of lithiasis smaller than $1 \mathrm{~cm}$ and 2.01 sessions in the group bigger than $1 \mathrm{~cm}$. The stones that required less number of sessions were located in the ureter and upper calyx. $71.2 \%$ of the patients were stone free after one treatment.

Conclusions: Siemens Lithostar Modularis has the adventages of a great confort of the patient during the procedure, better image because of a very good cuality of the fluoroscopy and the main point is a great comminution of the stone doing very easy the elimination of the fragments achieving a stone free rate of more than $90 \%$ in our experience, decreasing the need of auxiliary manoeuvres and the leng of the treatment.

Keywords: Lithiasis. Lithotripsy. ESWL. 
$\mathrm{D}$ esde el tratamiento del primer paciente en febrero de 1980 en Munich ${ }^{1}$ mediante litotricia extracorpórea por ondas de choque, esta técnica se ha convertido en el tratamiento estándar de la mayoría de las litiasis del aparato urinario. Tanto los litotritores como los tipos de energía han evolucionado desde el HM3 desarrollado por Dornier hasta la actualidad, existiendo varias generaciones de ellos, todos ellos con la finalidad última de destruir la litiasis urinaria con la mínima morbilidad. En la actualidad el tipo de energía considerado como el que mejor cumple estos criterios de mayor eficacia con la menor lesividad parece ser los generados por ondas electromagnéticas. Nuestro Hospital cuenta con litotricia extracorpórea desde 1989. En mayo de 2000 hemos adquirido el litotritor de Siemens Lithostar Modularis, presentando los resultados obtenidos con los primeros 1.000 pacientes tratados en nuestro Servicio.

\section{PACIENTES Y METODOS}

Presentamos el análisis retrospectivo de los datos referidos a 1.000 pacientes consecutivos con litiasis en el sistema urinario tratados desde junio de 2000 a diciembre de 2003 en la Unidad de Litotricia de la Obra Hospitalaria de Nuestra Señora de Regla de León. La litotricia extracorpórea se hizo con el litotritor Siemens Lithostar Modularis que consiste en un emisor de ondas electromagnéticas (Electromagnetic shock wave C plus), integrado a un arco fluoroscópico en $\mathrm{C}$ (Siremobil Iso-C) que permite la localización de las litiasis con un movimiento de eje de rayos $X$ de $-20^{\circ}$ a $+20^{\circ}$. Además tiene integrado un localizador ecográfico externo. También presenta la integración de una mesa urológica multifuncional Modularis Uro que facilita la aplicación de la litotricia. (Fig. 1)

A todos los pacientes se les hizo estudio preoperatorio y recibieron sedación endovenosa con alfentanilo y dormicum. Todos los pacientes se trataron de forma ambulatoria. El tamaño de la litiasis se determinó por el diámetro mayor de la litiasis, medido en una radiografía simple de abdomen previa a comenzar el tratamiento. La eficacia de la litotricia se evaluó mediante radiografía a la semana de la sesión de litotricia. La presencia de hematoma se evaluó mediante eco-

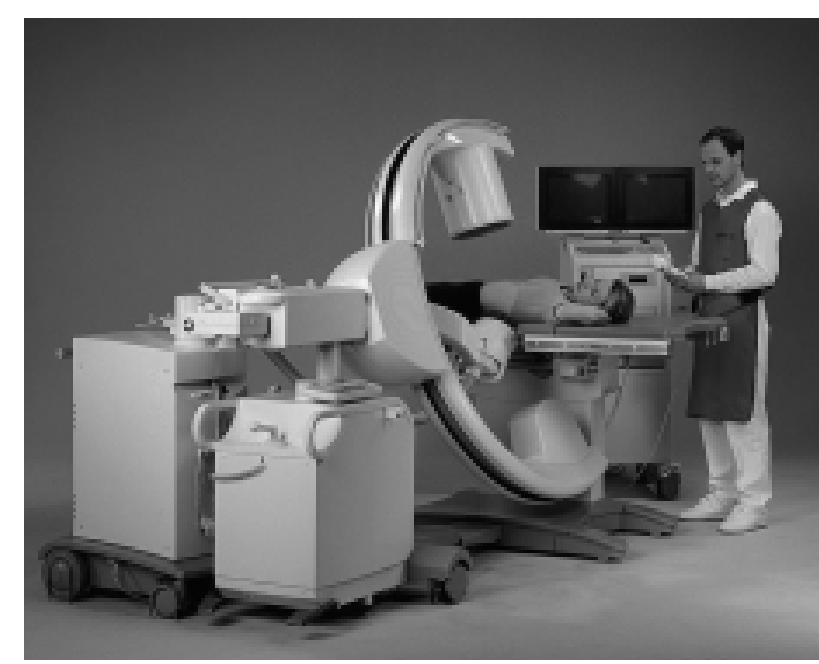

FIGURA 1: Imagen de litotritor Lithostar Modularis de Siemens: consta de generador de ondas, arco en C y mesa de tratamiento acopladas de forma sincrónica para el tratamiento.

grafía a la semana de la litotricia. El protocolo que usamos en nuestro servicio para el tratamiento de las litiasis es el tratamiento de las mismas in situ, aplicando el número de sesiones necesario hasta obtener fragmentos menores de $2 \mathrm{~mm}$ o la eliminación de la litiasis, momento en el que se les da el alta. Sólo se coloca catéter doble pig-tail o nefrostomía a posteriori de la litotricia, en los casos en los que existen complicaciones o que existe una obstrucción de la litiasis o en caso de impactación de las litiasis para su movilización. El tiempo medio de resolución de las litiasis, desde que son vistos por primera vez en el servicio hasta que son dados de alta, fue de 5 semanas. La aplicación de la energía empleada en las ondas de choque electromagnéticas depende de la localización de la litiasis (Fig. 2). La frecuencia de aplicación es de 60-90 ondas/minuto en las localizaciones en riñones y uréter proximal $\mathrm{y}$ de $60-120 / \mathrm{min}$ en las de uréter distal. El número medio de ondas por sesión fue de 3.000-3.500.

La localización de las litiasis fue en $44,1 \%$ derecha e izquierda en un $55,9 \%$ de los casos, $60,5 \%$ son varones y $39,5 \%$ mujeres. El $60,4 \%$ presentaban litiasis en el uréter y el $39,6 \%$ en la unidad renal. La edad media fue de 53,5 $\pm 14,62$ años (rango 21-84). El tamaño medio de las litiasis ha sido $1,22 \pm 0,74 \mathrm{~cm}$. (rango 0,4-8 cm). (Tabla 1). Un 80,8\% tenían una sola litiasis, el 10,8\% 2 litiasis y el 8,4\% tenían 3 o más litiasis. 


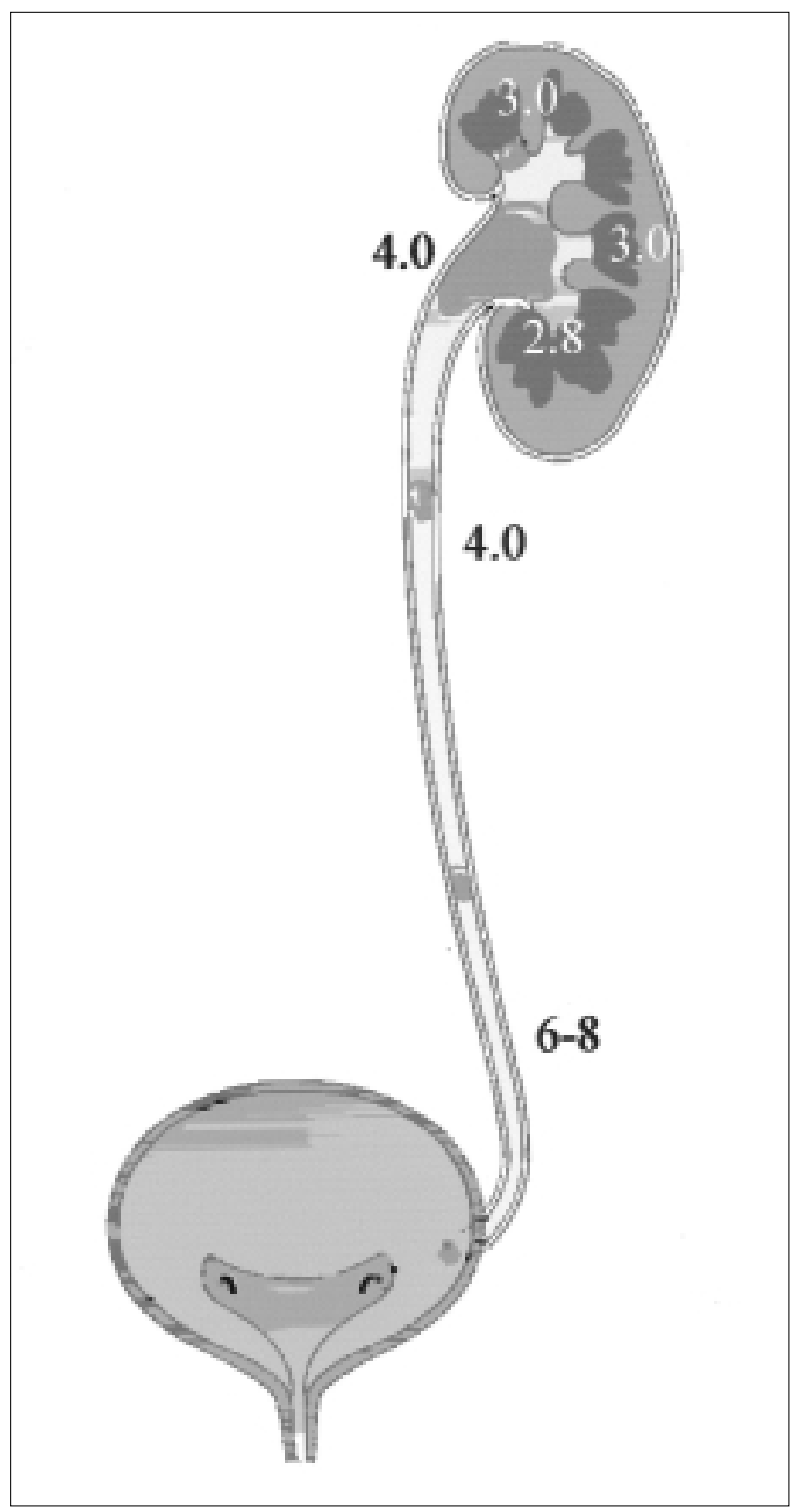

FIGURA 2: Niveles de energía empleados según la localización de la litiasis.
El número medio de sesiones por paciente fue de $1,6 \pm 1,13$. Si consideramos los pacientes con una sola litiasis, la media de sesiones de litotricia fue de $1,34+1,14$ sesiones por litiasis.

\section{RESULTADOS}

El número de sesiones fue menor en los de localización ureteral (1,57 por paciente) y dentro de estos los de localización en uréter lumbar, 1,38 sesiones. Coincide que el tamaño de las litiasis es también menor en los de localización ureteral, 0,99 cm de media frente a los de localización renal 1,55 $\mathrm{cm}$ de media (Tabla1).

Cuando separamos las litiasis por tamaños observamos que la media de sesiones es menor en las de menor tamaño, 1,32 sesiones en las menores de $1 \mathrm{~cm}$ frente a las 2,33 en las mayores de $2 \mathrm{~cm}$ (Tabla 2).

En un 21\% de los pacientes hubo que realizar maniobras instrumentales durante el tratamiento para resolver las litiasis o sus complicaciones. Entre ellas destacan las ureteroscopias que se llevaron a cabo en un $22 \%$ de las litiasis con localización en uréter pélvico. También destaca el 25\% de uso de catéteres en las litiasis localizadas en la pelvis renal (Tabla 3 ).

El 94\% de las litiasis se resolvieron sin restos, que corresponden a un 96\% en las de localización ureteral y a un 90\% de localización renal. En un $4 \%$ quedaron restos sedimentados y en un $2,5 \%$ no fue eficaz la litotricia. Destaca la no eficacia en las localizadas en uréter pélvico que alcanza el $7 \%$ y el 4,5\% en las localizadas en cáliz superior. El mayor porcentaje de restos sedimentados como era de esperar es en los pacientes en cáliz inferior con un 15\% (Tabla 4).

Tabla 1

Tamaño de las litiasis y sesiones de litotricia por localización

\begin{tabular}{|c|c|c|c|c|c|}
\hline Localización & Número (\%) & $\begin{array}{c}\text { Tamaño }(\mathrm{cm}) \\
\text { media } \pm \text { desviación estándar }\end{array}$ & Rango (cm) & $\begin{array}{c}\text { Sesiones } \\
\text { media } \pm \text { desviación estándar }\end{array}$ & Rango \\
\hline Uréter lumbar & $293(48,5 \%)$ & $0,99 \pm 0,37$ & $0,4-2,5$ & $1,38 \pm 0,77$ & $1-7$ \\
\hline Uréter iliaco & $52(8,6 \%)$ & $1,14 \pm 0,23$ & 0,72 & $1,75 \pm 1,20$ & $1-5$ \\
\hline Uréter pélvico & 259 (42,9\%) & $0,95 \pm 0,41$ & 0,53 & $1,75 \pm 1,12$ & $1-8$ \\
\hline Total uréter & 604 & $0,99 \pm 0,38$ & $0,4-2,6$ & $1,57 \pm 0,99$ & $1-8$ \\
\hline Cáliz superior & $32(8,1 \%)$ & $1,14 \pm 0,47$ & $0,3-2,3$ & $1,59 \pm 1,40$ & $1-7$ \\
\hline Cáliz medio & $32(8,1 \%)$ & $1,10 \pm 0,39$ & 0,52 & $1,68 \pm 1,08$ & $1-4$ \\
\hline Cáliz inferior & $121(30,6 \%)$ & $1,25 \pm 0,60$ & 0,54 & $1,48 \pm 0,75$ & $1-4$ \\
\hline Pelvis & $211(53,3 \%)$ & $1,85 \pm 1,15$ & 0,58 & $2,02 \pm 1,49$ & $1-9$ \\
\hline Total renales & 396 & $1,55 \pm 0,97$ & $0,5-8$ & $1,80 \pm 1,30$ & $1-9$ \\
\hline Total & 1000 & $1,21 \pm 0,73$ & $0,4-8$ & $1,66 \pm 1,35$ & $1-9$ \\
\hline
\end{tabular}


Tabla 2

Sesiones de litotricia por diferentes tamaños

\begin{tabular}{lccc}
\hline Tamaño & Número & $\begin{array}{c}\text { No de sesiones } \\
\text { media } \pm \text { desviación } \\
\text { estándar }\end{array}$ & Rango \\
\hline $0,4<1 \mathrm{~cm}$ & 221 & $1,32 \pm 0,63$ & $1-4$ \\
$0,4-1 \mathrm{~cm}$ & 591 & $1,41 \pm 0,78$ & $1-7$ \\
$1 \mathrm{~cm}$ & 370 & $1,47 \pm 0,85$ & $1-7$ \\
$>1 \mathrm{~cm}$ & 409 & $2,01 \pm 1,43$ & $1-9$ \\
$>1<2 \mathrm{~cm}$ & 277 & $1,86 \pm 1,25$ & $1-7$ \\
$\geq 2 \mathrm{~cm}$ & 132 & $2,33 \pm 1,72$ & $1-9$ \\
Total & 1000 & $1,66 \pm 1,13$ & $1-9$ \\
\hline
\end{tabular}

Quedaron libres de litiasis tras una sola sesión de litotricia el $76 \%$ de los pacientes con litiasis menores de $1 \mathrm{~cm}$, en los mayores de $2 \mathrm{~cm}$ este porcentaje fue del $38 \%$. Del total de pacientes con una sola litiasis con independencia del tamaño de la misma, quedaron libres de litiasis en una sola sesión el $80 \%$.

\section{DISCUSIÓN}

Las ventajas del tratamiento con litotricia extracorpórea son la posibilidad de tratar el paciente de forma ambulatoria, con una técnica de alta eficacia y baja morbilidad. La litotricia extracorpórea se considera de primera elección en la mayoría de las litiasis del sistema urinario ${ }^{2}$.

El uso de un generador de ondas electromagnéticas está desplazando al resto de tecnologias, debido a que conjuga una alta eficacia con una baja morbilidad. Los generadores electrohidraúlicos están en desuso debido a la alta morbilidad y

Tabla 3

Maniobras complementarias a la litotricia dependiendo de la localización

\begin{tabular}{lccccc}
\hline Localización & Maniobras & Stent & URS & Nefrostomía & Ureterolitotomía \\
\hline Uréter lumbar & $15 \%$ & $10,5 \%$ & $4 \%$ & $2,5 \%$ & \\
Uréter iliaco & $11 \%$ & $8,3 \%$ & $3 \%$ & $3 \%$ & \\
Uréter pélvico & $30 \%$ & $9,5 \%$ & $22 \%$ & $4 \%$ & $1 \%$ \\
Total Uréter & $21,2 \%$ & $9,2 \%$ & $11,6 \%$ & $3 \%$ & $0,5 \%$ \\
Cáliz superior & $9 \%$ & & $9 \%$ & & \\
Cáliz medio & $0 \%$ & & & & \\
Cáliz inferior & $5 \%$ & $5 \%$ & & & $0,6 \%$ \\
Pelvis renal & $32,5 \%$ & $25,2 \%$ & $4 \%$ & $4,7 \%$ & $0,4 \%$ \\
Total renal & $20,6 \%$ & $16 \%$ & $3 \%$ & $2,5 \%$ & \\
TOTAL & $20,9 \%$ & $11,8 \%$ & $8,1 \%$ & $3 \%$ & $0,4 \%$ \\
\hline
\end{tabular}

la escasa aportación en eficacia respecto a las ondas electromagnéticas ${ }^{3}$. Los piezoeléctricos aunque de baja morbilidad pecan de menos eficacia que las ondas electromagnéticas ${ }^{4}$.

La media de sesiones de litotricia para resolver la litiasis ha sido de 1,6. Cuando consideramos solamente las litiasis únicas esta disminuye al 1,3 , lo que sería un mejor indicador ya que estaríamos hablando de sesiones de litotricia por unidad de litiasis.

Al igual que la mayoría de los estudios el tamaño influye en el número de sesiones que se precisan, pasando de 1,3 sesiones en los pacientes con litiasis menores de $1 \mathrm{~cm}$ a 2,3 sesiones en pacientes con litiasis mayores de $2 \mathrm{~cm}$.

Cuando consideramos la localización de la litiasis, es menor el número de sesiones necesarias para resolver las litiasis en las de localización ureteral, 1,57 sesiones frente a las 1,8 renales, pero hemos de indicar que el tamaño medio de las litiasis es menor en las ureterales, $0,99 \mathrm{~cm}$ frente a los $1,55 \mathrm{~cm}$, pudiendo deberse la diferencia única y exclusivamente a que las de localización ureteral son más pequeñas y como hemos indicado antes se ha comprobado que a menor tamaño menor número de sesiones.

Los pacientes precisan en un $21 \%$ de los casos maniobras complementarias para la resolución de las litiasis. Destacar que el $32,5 \%$ de las localizadas a nivel de pelvis renal, asociado principalmente a su tamaño, precisan de la colocación de un catéter tipo doble pig-tail para favorecer la eliminación de fragmentos en las litiasis mayores de $2 \mathrm{~cm}$ y también se asocian a complicaciones como son la obstrucción y/o infección, colocando en este caso catéteres tipo doble pig-tail y nefrostomías. También destaca el $30 \%$ de maniobras complementarias en los pacientes con litiasis localizada en el uréter pélvico, sobre todo ureteroscopia, debido al enclavamiento de la litiasis o a restos de otras litiasis.

La eficacia en la resolución de las litiasis, es máxima en la localización de uréter iliaco, la litotricia es más resolutiva a nivel ureteral (96\%) que a nivel renal (90\%). En la localización 
Tabla 4

Eficacia de la litotricia dependiendo de la localización de la litiasis

\begin{tabular}{lccc}
\hline Localización & No eficaz & Sedimentado & No restos \\
\hline Uréter lumbar & $1,5 \%$ & & $98,5 \%$ \\
Uréter iliaco & & & $100 \%$ \\
Uréter pélvico & $7 \%$ & & $93 \%$ \\
Total Uréter & $3,9 \%$ & & $96,1 \%$ \\
Cáliz superior & $4,5 \%$ & $4,5 \%$ & $91 \%$ \\
Cáliz medio & & $13,6 \%$ & $86,4 \%$ \\
Cáliz inferior & & $15 \%$ & $85 \%$ \\
Pelvis renal & $0,4 \%$ & $6,6 \%$ & $93,4 \%$ \\
Total renal & & $9,4 \%$ & $90,3 \%$ \\
Total & $2,5 \%$ & $3,7 \%$ & $94 \%$ \\
\hline
\end{tabular}

en la que la ausencia de eficacia de fragmentación es más alta es a nivel de uréter pélvico, principalmente en las litiasis impactadas. El mayor número de restos de litiasis sedimentados es en el cáliz inferior como era de esperar.

\section{CONCLUSIONES}

La media de sesiones de litotricia en los pacientes con una sola litiasis fue de 1,3 sesiones por litiasis, en el conjunto de pacientes con independencia del número de litiasis esta fue de 1,6. El tamaño está relacionado de forma directa con el número de sesiones, a mayor tamaño, mayor número de sesiones. En las litiasis menores de $1 \mathrm{~cm}$ ésta desapareció en una sola sesión en un $76 \%$ de los pacientes, mientras que en las litiasis mayores de $2 \mathrm{~cm}$ esto sucede solamente en un $38 \%$ de los pacientes. Por localización en los que se necesitó menor número de sesiones fue en los ureterales coincidiendo que son también los de menor tamaño. El 21\% de los pacientes precisaron maniobras instrumentales complementarias para la eliminación de las litiasis. La resolución de las litiasis, con la eliminación completa de las mismas se produjo en un $94 \%$ de los pacientes. De todo lo anterior podemos deducir que Lithostar Modularis de Siemens es un instrumento altamente eficaz para el tratamiento con éxito de las litiasis urinarias. Las ventajas de este litotritor, además de los adelantos técnicos tanto en comodidad del paciente, localización de la litiasis y mejor calidad de la escopia, se deben sobre todo a su alta eficacia, con una fragmentación de la litiasis en pequeños fragmentos, facilitando su eliminación, consiguiendo una resolución sin restos de litiasis por encima del $90 \%$. Además disminuye la necesidad de maniobras complementarias y acorta el tiempo de tratamiento por la fácil eliminación de los restos fragmentados.

\section{REFERENCIAS}

1. Chaussy $\mathrm{CH}$, Brendel W, Schmiedt E. Extracorporeally induced destruction of kidney stones by shock waves. Lancet 1980; 13:1265-1268.

2. Tiselius HG, Ackerman D, Alken G, Buck C, Conort P. Galluci M. Guidelines on urolithiasis. Eur Urol 2001;40: 362-371.

3. Tailly, GG. In situ SWL of ureteric stones: comparison between electrohydraulic and electromagnetic shock wave sources. J Endourol 2002; 16:209-2 14.

4. Robert M, Rakotomalala E, Delbos O, Navratl H. Piezoelectric Lithotripsy of Ureteral Sones: Influence of Shockwave frecuence on sedation and therapeutic efficiency. J. Endourol. 1999; 13: 157-160.

Dr. A. Vega Vega

Gran Vía de San Marcos 42; 6-B

24002 León

(Trabajo recibido el 31 enero de 2005) 